\title{
Nanoscale Imaging of Photonic Densities of States in Finite Photonic Structures
}

\author{
Judy J. Cha*, Eric Smith**, Martin Couillard*†, David A. Muller* \\ * School of Applied and Engineering Physics, Cornell University, Ithaca, NY 14853 \\ ** Department of Physics, Columbia University, New York, NY 10027 \\ $\uparrow$ Current address: Semiconductor Insights, Ottawa, ON, Canada K2K 2X2
}

Relativistic electrons passing in or near a dielectric medium generate Cherenkov and transition radiations which act as a virtual light source, coupling to photonic densities of states (DOS) [1].

| Electron energy-loss spectroscopy (EELS) can thus be used as a local probe of the optical modes of dielectric structures whose features are in nanoscale in a spectral domain ranging from the ultraviolet to the near-infrared [2]. Due to recent developments of monochromators and microscope stability, energy resolution of EELS has improved to reach $100 \mathrm{meV}$ which is comparable to $\sim 30 \mathrm{~nm}$ spectral resolution in the visible frequencies, making EELS an attractive complimentary technique to opticsbased techniques for studying photonic structures with a superior spatial resolution. An important benefit of EELS is the ability to probe the high-wavelength optical domain, including the telecom wavelength of $1.5 \mu \mathrm{m}$, in addition to a broad spectral range extending down to x-ray wavelengths. Using spectroscopic imaging (SI) [3], spatial distributions of photonic modes can also be mapped.

We probed local photonic DOS of diamond and silicon photonic structures of finite sizes, using monochromated EELS acquired with a sub-5nm electron probe at $\sim 130 \mathrm{meV}$ energy resolution.

| The structures were fabricated using a focused ion beam, milling periodic patterns across a few microns onto the dielectric slabs. The observed photonic modes in EELS were compared with simulated spectra using a local, relativistic dielectric theory [4] and the MIT PB package [5].

Figure 1a shows that radiation is induced in a $2-\mu \mathrm{m}$ thick diamond film, indicated by the noticeable intensity in EELS below $5.5 \mathrm{eV}$, the electronic band gap of diamond. When boundary conditions are imposed such as the waveguide shown in Fig. $1 \mathrm{~b}$ inset, peaks emerge in EELS, which agrees with the simulated spectrum using a relativistic dielectric theory (Fig. 1b). Figure 2a-c show silicon photonic structures of cylindrical holes patterned in a hexagonal lattice grid. The radius, $r$, of the cylindrical holes was varied while keeping the lattice constant, $a$, constant in order to observe how the photonic modes would shift in energy with varying $r / a$ ratios. Figure $2 \mathrm{~d}$ shows EELS for the $r / a$ $=0.34$ case. The experimentally observed peaks match well with the simulated photonic DOS of the same structure. Two extra peaks that appear in the simulated DOS are not resolved in the experiment due to the low energy resolution of $\sim 100 \mathrm{meV}$. When the photonic DOS is sampled at the experimental energy resolution, the extra peaks damp out. Figure 2e shows how the modes shift in their energies with varying $r / a$ ratios. Using EEL-SI, photonic modes in a silicon structure of rectangular holes patterned in a square lattice, shown in Fig. 3a, are spatially mapped. The areas of the two modes, indicated by arrows in Fig. 3b, were integrated to create intensity maps which are shown in Fig. $3 \mathrm{c}$ and $\mathrm{d}$. The two modes reflect the corner and side symmetry of the photonic structure. The averaged line profiles from the intensity maps are shown in Fig. 3e and $\mathrm{f}$.

\section{References:}

[1] F. J. Garcia de Abajo and M. Kociak, Phys. Rev. Lett. 100, 106804 (2008).

[2] A. Yurtsever et al., Phys. Rev. Lett. 100, 217402 (2008). 
[3] J. Nelayah et al., Nat. Phys. 3, 348 (2007); D. A. Muller et al., Science 319, 1073 (2008).

[4] J. P. R. Bolton and M. Chen, Ultramicroscopy 60, 247 (1995).

[5] S. G. Johnson and J. D. Joannopoulos, Optics Express 8, 173 (2001).

[6] Supported by CCMR, an NSF MRSEC (DMR 0520404) and NSF EEC-0117770, 0646547.
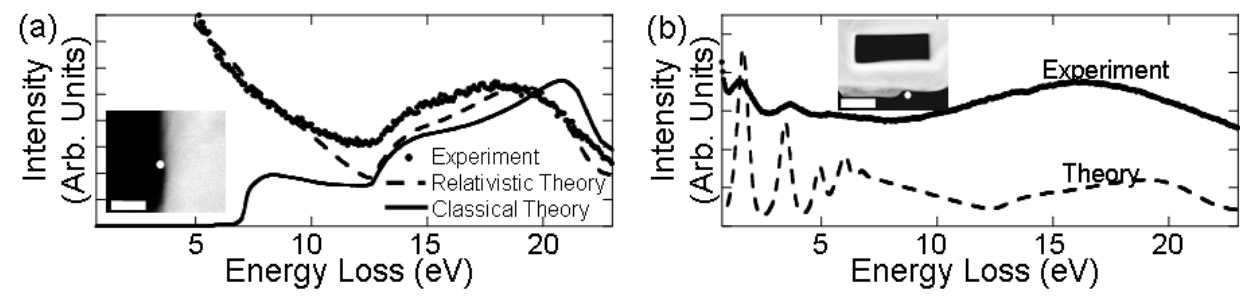

Figure 1. EELS near a diamond film and a waveguide. (a) Radiation is induced as swift electrons pass near the film. (b) When boundary conditions are imposed (inset), peaks emerge, indicating those modes whose frequencies are supported by the boundary conditions. Scale bars $=200 \mathrm{~nm}$.

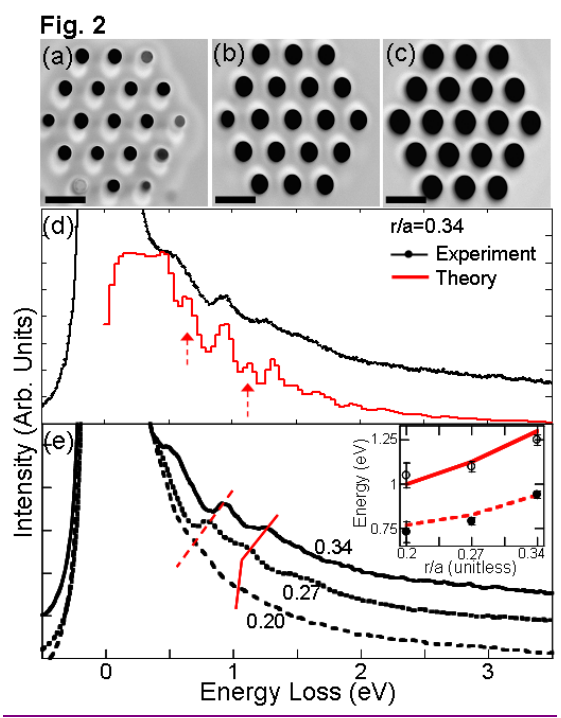

Fig. 3

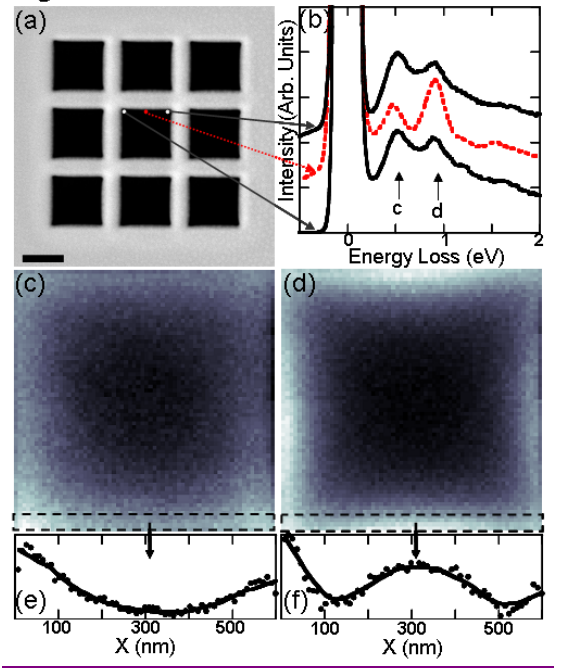

Formatted Table

Formatted: Left 


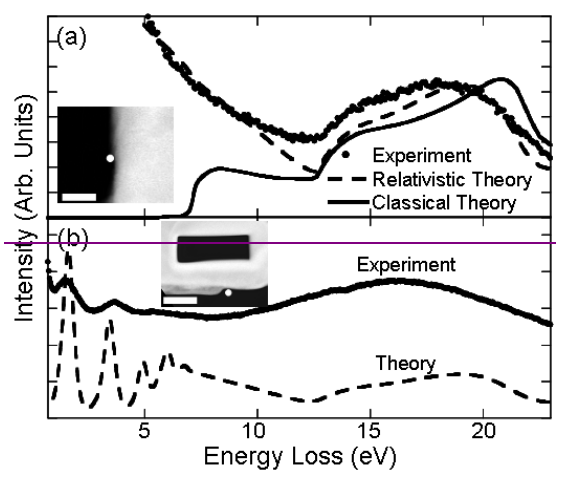

Figure 1. EEL spectra near diamond film and waveguide. (a) Radiation is induced as swift electrons pass near the diamond film. (b) When boundary conditions are imposed as shown in the inset, peaks emerge in EELS, indicating the modes whose frequencies are supported by the boundary conditions. Seale bars $=200 \mathrm{~nm}$.

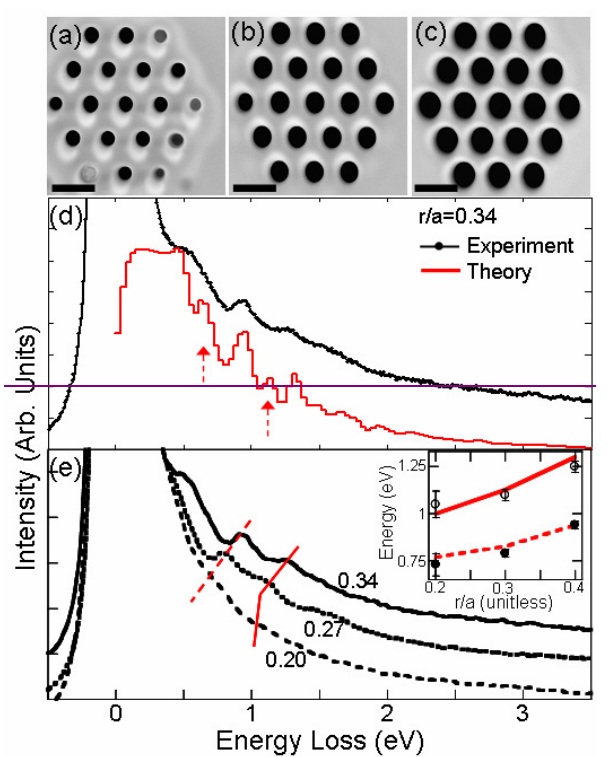

Figure 2. Silicon photonic structures of cylindrical holes on a hexagonal lattice grid with varying r/a ratios: 0.20 in (a), 0.27 in (b), and 0.34 in (c). (d) shows the observed photonic modes in EELS and the simulated photonic DOS for the $\mathrm{r} / \mathrm{a}=0.34$ case. (e) Shifts in the photonic mode energies were observed with varying r/a ratios. The inset shows the agreement between the experiment and

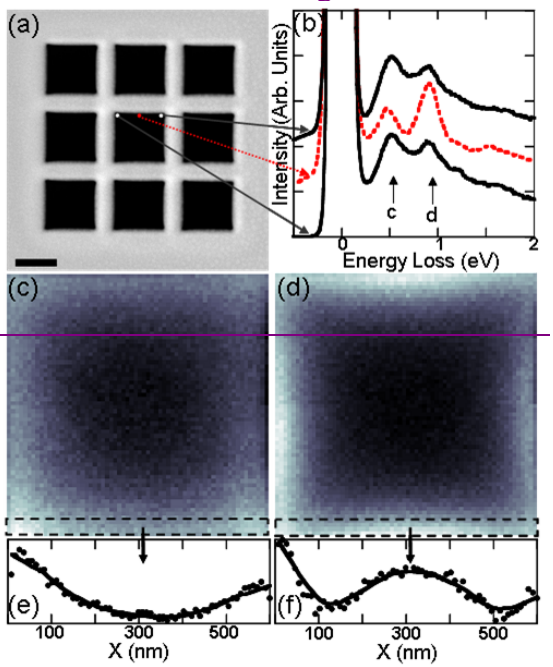

the simulation. Scale bars $=500 \mathrm{~nm}$.

Figure 2 (above). Silicon photonic structures of cylindrical holes on a hexagonal lattice grid with varying r/a ratios: 0.20 in (a), 0.27 in (b), and 0.34 in (c). (d) shows the observed photonic modes in EELS and the simulated photonic DOS for the $\mathrm{r} / \mathrm{a}=0.34$ case. (e) Shifts in the photonic mode energies were observed with varying $\mathrm{r} / \mathrm{a}$ ratios. The inset shows the agreement between the 
experiment and the simulation. Seale bars $=500 \mathrm{~nm}$.

Figure 3 (left). Mapping of the spatial distributions of photonic modes in a structure of hollow squares on a square lattice grid. Spectra near the top side of the square are selectively shown in (b). The areas of the two peaks, indicated by the arrows, are integrated to obtain intensity maps of the central square, which

Figure 3. Mapping of the spatial distributions of photonic modes in a structure of hollow squares on a square lattice grid. Spectra near the top side of the square are selectively shown in (b). The areas of the two peaks, indicated by the arrows, are integrated to obtain intensity maps of the central square, which show that the two peaks correspond to a corner and a side mode respectively, as shown in (c) and (d). (e) and (f) show averaged line profiles obtained from (c) and (d). Scale bars $=500 \mathrm{~nm}$.

show that the two peaks correspond to a corner and a side mode respectively, as shown in (c) and (d). (e) and (f) show averaged line profiles obtained from (c) and (d). Seale bars $=500 \mathrm{~nm}$. 